\title{
A MEAN-FIELD MODEL FOR SPIN DYNAMICS IN MULTILAYERED FERROMAGNETIC MEDIA
}

\author{
JINGRUN CHEN, CARLOS J. GARCÍA-CERVERA, AND XU YANG
}

\begin{abstract}
In this paper, we develop a mean-field model for describing the dynamics of spin transfer torque in multilayered ferromagnetic media. Specifically, we use the techniques of Wigner transform and moment closure to connect the underlying physics at different scales, and reach a macroscopic model for the dynamics of spin coupled with the magnetization within the material. This provides a further understanding of the linear response model proposed by Zhang, Levy, and Fert in [42], and in particular we get an extra relaxation term which helps to stabilize the system. We develop efficient numerical methods to overcome the stiffness appearing in this new mean-field model, and present several examples to analyze and show its validity.
\end{abstract}

\section{INTRODUCTION}

Spintronics, i.e., the active control and manipulation of spin degrees of freedom, plays a key role in advancing technologies and the design of new electronic devices. The discovery of the Giant Magnetoresistance effect (GMR) by A. Fert and P. Grünberg [5, 21] has enabled the development of new technologies. This phenomenon has been observed in magnetic multilayered media (e.g., [5]), and has been used to detect domain-wall motion ([32]), a mechanism that is important in operating spintronic memories and logic devices $([15])$. When an electron current flows in a ferromagnetic material, conservation of spin yields a transfer of spin angular momentum to the magnetization, an effect known as spin-transfer torque (STT), and this has been used in technological applications such as the magnetoresistance random access memories (MRAMs), race-track memories (e.g., [9]), and the control of the dynamics of domain walls $(e . g .,[31,15])$. In this article we are primarily interested in the magnetization reversal process in ferromagnetic media as a result of this spin-transfer torque (see Figure 1).

The magnetization reversal process in a spintronic device involves a multitude of physical processes, including spin polarization, spin transport and diffusion within ferromagnetic multilayers, and spin-magnetization interactions (e.g., $[24,35,9])$. Experiments have been done in a large number

Date: February 4, 2015.

2000 Mathematics Subject Classification. 65M06, 65Z05, 74F15, 78A25, 82D40.

Key words and phrases. spin-transfer torque, spin-magnetization coupling, moment closure, magnetization reversal. 
of magnetic materials, ranging from metals (e.g., $\mathrm{Fe}, \mathrm{Co}, \mathrm{Ni},[24,9])$ and their alloys to semiconductors (e.g., diamond and organic semiconductors, $[22,36])$. The first models for STT were developed independently by Berger and Slonczewski $([8,38])$. More recent results can be characterized into two groups, according to whether the spin dynamics is included in the model or not: In $[43,40,13]$, the magnetization dynamics is considered with the STT term being described as external torques depending on the magnetization and its derivatives; while in $[42,34]$, the magnetization dynamics is coupled to the spin dynamics on an equal footing. Under the assumption that spatial variation of the spin is neglectable, the coupled system can be reduced to the magnetization dynamics with additional torque terms [43]. However, this assumption may not be true in general and the coupled system is highly nonlinear, hence the representability of spin dynamics in terms of magnetization-related torques is not guaranteed.

In the STT context, very few attempts have been made to describe the spin dynamics at the microscopic level, with the exception of [13, 34]. Only the magnetization dynamics was considered in [13], with additional torques derived from a microscopic model. In [34], the authors derived the spin dynamics from a kinetic model, but failed to recover the spin dynamics obtained from the linear response theory [42]. These issues are our main motivation to study the connection between models of spin dynamics at different physical scales, and to derive mean-field models for the spin dynamics coupled to magnetization dynamics.

The dynamics of the magnetization is typically described by the LandauLifshitz-Gilbert (LLG) equation [26, 19]. To describe the electron transport, we start from a Schrödinger equation in the spinor form, and use the wigner transform to obtain a kinetic model. From this, we obtain a mean-field model by carrying on a moment closure approximation of the kinetic model, including Bloch collision terms. To compare the mean-field model with the one in [42], we restrict ourselves to the case of metals by applying the quasi-static approximations on the spin current, in which an additional term that stabilizes the system is obtained. Under the assumption that the spinmagnetization coupling is weak, the mean-field model recovers the model in [42]. We develop a time-splitting computational method to solve the equation of spin dynamics, and use the Gauss-Seidel projection method [41] to solve the magnetization dynamics. We illustrate the magnetization reversal process with several examples.

The paper is organized as follows: In Section 2, we derive the mean-field model by applying the Wigner transform on the spinor dynamics, and the moment closure on the resulting kinetic equations with Bloch collision terms; we also compare it to the one in [42]. We describe the numerical methods for solving the mean-field model coupled to LLG in Section 3, and present several examples on magnetization switching in ferromagnetic multilayer in Section 4. Conclusive remarks are made in Section 5. 


\section{A MEAN-FIELD MODEL FOR SPIN DYNAMICS}

Figure 1 is the standard device for ferromagnetic multilayers, where two ferromagnetic layers (FM1 and FM2) are sandwiched by a nonmagnetic metallic spacer (NM). Denote domains occupied FM1, FM2 and NM by $\Omega_{\mathrm{FM} 1}, \Omega_{\mathrm{FM} 2}$ and $\Omega_{\mathrm{NM}}$ with the thickness $D_{1}, D_{2}$ and $D_{3}$, respectively. Let $\Sigma=\Omega_{\mathrm{FM} 1} \cup \Omega_{\mathrm{FM} 2}$ and $\Omega=\Sigma \cup \Omega_{\mathrm{NM}}$. Spin currents are applied perpendicular to the device to make ease of magnetization switching. In such a system, there are two types of electrons essentially $[43,33]$ : one is below the Fermi level, which is responsible for magnetization dynamics and the other is around the Fermi sea, which is responsible for spin dynamics. It is impossible to unambiguously separate these two types of electrons, so a surrogate "s-d" Hamiltonian is employed to describe the spin-magnetization interaction.

2.1. Spin dynamics. In this section, we drive the mean-field model for spin dynamics. We first start with the spinor dynamics in quantum mechanics.

2.1.1. From one-body Schrödinger equation to kinetic description. A simple model for spin dynamics is given by the following one-body Schrödinger equation

$$
\mathrm{i} \hbar \frac{\partial}{\partial t} \boldsymbol{\psi}(\boldsymbol{x}, t)=\mathcal{H}(\boldsymbol{x}, t) \boldsymbol{\psi}(\boldsymbol{x}, t),
$$

where $\boldsymbol{\psi}=\left(\psi_{+}, \psi_{-}\right)^{T}$ is called the spinor, and the effective one-body Hamiltonian is of the form [33]

$$
\mathcal{H}(\boldsymbol{x}, t)=\left(-\frac{\hbar^{2}}{2 m} \nabla_{\boldsymbol{x}}^{2}+V(\boldsymbol{x})\right) \mathrm{I}-\frac{J}{2} \hat{\boldsymbol{\sigma}} \cdot \boldsymbol{m}(\boldsymbol{x}, t),
$$

where $\hbar$ is the rescaled Planck constant, $m$ is the effective mass of an electron, $V(\boldsymbol{x})$ is the external potential, I is the identity matrix, $J$ is the spinmagnetization coupling constant, and $\boldsymbol{m}$ is the background magnetization. The Pauli matrices $\hat{\boldsymbol{\sigma}}=\sigma_{x} \hat{\boldsymbol{i}}+\sigma_{y} \hat{\boldsymbol{j}}+\sigma_{z} \hat{\boldsymbol{k}}$ where

$$
\sigma_{x}=\left(\begin{array}{cc}
0 & 1 \\
1 & 0
\end{array}\right), \quad \sigma_{y}=\left(\begin{array}{cc}
0 & -i \\
i & 0
\end{array}\right), \quad \sigma_{z}=\left(\begin{array}{cc}
1 & 0 \\
0 & -1
\end{array}\right) .
$$

The last term is the "s-d" Hamiltonian, which mimics the spin-magnetization interaction. The "s-d" Hamiltonian is obtained by neglecting the self-induced electromagnetic fields generated by the conduction electrons. It seems in the community of spintronics that this is an adequate approximation, e.g., [43, $33]$.

Next we give a kinetic description of spinor dynamics via the following Wigner transform of $\boldsymbol{\psi}$,

$$
W(\boldsymbol{x}, \boldsymbol{v}, t)=\left(\frac{m}{2 \pi \hbar}\right)^{3} \int_{\mathbb{R}^{3}} \boldsymbol{\psi}\left(\boldsymbol{x}-\frac{\boldsymbol{y}}{2}, t\right) \otimes \boldsymbol{\psi}^{*}\left(\boldsymbol{x}+\frac{\boldsymbol{y}}{2}, t\right) \mathrm{e}^{\mathrm{i} \frac{m}{\hbar} \boldsymbol{v} \cdot \boldsymbol{y}} \mathrm{d} \boldsymbol{y},
$$

where the Wigner function $W(\boldsymbol{x}, \boldsymbol{v}, t)$ is a $2 \times 2$ matrix function, and $\boldsymbol{\psi}^{*}$ is the complex conjugate transpose of $\boldsymbol{\psi}$. For simplicity, the Wigner transform 
(3) is defined using a single wave function. Due to the linearity of the Schrödinger equation, the normalization constant for the wave function does not affect the derived model. Meanwhile, the model can be modified to take into account either finitely many or infinitely many wave functions; see [29] for example. We stress that all these will not change the models derived in the current work.

$W(\boldsymbol{x}, \boldsymbol{v}, t)$ is connected to the macroscopic quantities via its moments:

$$
\begin{aligned}
& \text { the charge density: } \quad n(\boldsymbol{x}, t)=\int_{\mathbb{R}^{3}} \operatorname{Tr}(W) \mathrm{d} \boldsymbol{v}, \\
& \text { the charge current: } \quad \boldsymbol{j}_{n}(\boldsymbol{x}, t)=\int_{\mathbb{R}^{3}} \boldsymbol{v} \operatorname{Tr}(W) \mathrm{d} \boldsymbol{v}, \\
& \text { the spin density: } \quad \boldsymbol{s}(\boldsymbol{x}, t)=\int_{\mathbb{R}^{3}} \operatorname{Tr}(\hat{\boldsymbol{\sigma}} W) \mathrm{d} \boldsymbol{v}, \\
& \text { the spin current: } \quad J_{\boldsymbol{s}}(\boldsymbol{x}, t)=\int_{\mathbb{R}^{3}} \boldsymbol{v} \otimes \operatorname{Tr}(\hat{\boldsymbol{\sigma}} W) \mathrm{d} \boldsymbol{v} .
\end{aligned}
$$

They are scalar, vector, vector, and matrix functions of $\boldsymbol{x}$ and $t$, respectively. Here Tr represents the trace operator over the spin space. Differentiating (3) with respect to $t$ yields

$$
\begin{array}{r}
\mathrm{i} \hbar \partial_{t} W(\boldsymbol{x}, \boldsymbol{v}, t)=\left(\frac{m}{2 \pi \hbar}\right)^{3} \int_{\mathbb{R}^{3}}\left[\left(\mathrm{i} \hbar \partial_{t} \boldsymbol{\psi}\left(\boldsymbol{x}-\frac{\boldsymbol{y}}{2}, t\right)\right) \otimes \boldsymbol{\psi}^{*}\left(\boldsymbol{x}+\frac{\boldsymbol{y}}{2}, t\right) \mathrm{e}^{\mathrm{i} \frac{m}{\hbar} \boldsymbol{v} \cdot \boldsymbol{y}}\right. \\
\left.+\boldsymbol{\psi}\left(\boldsymbol{x}-\frac{\boldsymbol{y}}{2}, t\right) \otimes\left(\mathrm{i} \hbar \partial_{t} \boldsymbol{\psi}^{*}\left(\boldsymbol{x}+\frac{\boldsymbol{y}}{2}, t\right)\right) \mathrm{e}^{\mathrm{i} \frac{m}{\hbar} \boldsymbol{v} \cdot \boldsymbol{y}}\right] \mathrm{d} \boldsymbol{y} .
\end{array}
$$

After some calculations (provided in Appendix A), one can reach the following Liouville equation from (1),

$$
\begin{aligned}
\partial_{t} W(\boldsymbol{x}, \boldsymbol{v}, t)+\boldsymbol{v} \cdot \nabla_{\boldsymbol{x}} W(\boldsymbol{x}, \boldsymbol{v}, t) & -\frac{e}{m} \boldsymbol{E} \cdot \nabla_{\boldsymbol{v}} W(\boldsymbol{x}, \boldsymbol{v}, t) \\
& -\frac{\mathrm{i}}{2 \hbar}[J \hat{\boldsymbol{\sigma}} \cdot \boldsymbol{m}(\boldsymbol{x}, t), W(\boldsymbol{x}, \boldsymbol{v}, t)]=0,
\end{aligned}
$$

where $e$ is the electron charge and $\boldsymbol{E}=\nabla V / e$.

In general $\hbar$ is a small parameter, which would naively indicate that the last left-hand term of (9) is the dominating one. However, the last two terms are actually comparable if one pays careful attention to their orders in physical units,

$$
\begin{aligned}
\frac{e}{m} \frac{|\boldsymbol{E}|}{|\boldsymbol{v}|} & \approx \frac{1.6 \times 10^{-19} \times 10^{7}}{9.109 \times 10^{-31} \times 10^{4}} \approx 1.757 \times 10^{14} \mathrm{~s}^{-1}, \\
\frac{J}{\hbar} & \approx \frac{1.602 \times 10^{-20}}{1.055 \times 10^{-34}} \approx 1.518 \times 10^{14} \mathrm{~s}^{-1},
\end{aligned}
$$

where the physical constants are $e=1.6 \times 10^{-19} \mathrm{C}, \hbar=1.055 \times 10^{-34} \mathrm{~J} \cdot \mathrm{s}$ and $m \sim m_{\mathrm{e}}=9.109 \times 10^{-31} \mathrm{~kg}$. For the device we considered here, typically $|\boldsymbol{E}| \approx 10^{5} \sim 10^{7} \mathrm{~V} / \mathrm{m},|\boldsymbol{v}| \approx 10^{4} \sim 10^{6} \mathrm{~m} / \mathrm{s}$, and $J \approx 1.602 \times 10^{-20} \mathrm{~J}(0.1 \mathrm{eV})$. 
2.1.2. From the kinetic model to the mean-field model and its hydrodynamic limit. In order to derive the mean-field model, we need to add collision terms in (9). For simplicity we consider the s-wave form used in the physical studies, e.g., [34],

$$
\begin{aligned}
\partial_{t} W(\boldsymbol{x}, \boldsymbol{v}, t) & +\boldsymbol{v} \cdot \nabla_{\boldsymbol{x}} W(\boldsymbol{x}, \boldsymbol{v}, t)-\frac{e}{m} \boldsymbol{E} \cdot \nabla_{\boldsymbol{v}} W(\boldsymbol{x}, \boldsymbol{v}, t) \\
& -\frac{\mathrm{i}}{2 \hbar}[J \hat{\boldsymbol{\sigma}} \cdot \boldsymbol{m}(\boldsymbol{x}, t), W(\boldsymbol{x}, \boldsymbol{v}, t)]=-\left(\frac{\partial W(\boldsymbol{x}, \boldsymbol{v}, t)}{\partial t}\right)_{\mathrm{colli}},
\end{aligned}
$$

where

$$
\left(\frac{\partial W}{\partial t}\right)_{\text {colli }}=\frac{W-\bar{W}}{\tau}+\frac{2}{\tau_{\mathrm{sf}}}\left(\bar{W}-\frac{I}{2} \operatorname{Tr} \bar{W}\right)
$$

Here $\bar{W}=\frac{1}{4 \pi} \int \mathrm{d} \Omega_{\boldsymbol{v}} W(\boldsymbol{x}, \boldsymbol{v}, t)$ is the angular average over the $\boldsymbol{v}$ space. Since an electron carries both charge and spin, we describe the no-spin-flip collision and the spin-flip collision by momentum-independent relaxation time approximations with the characteristic time scale of electron collision $\tau$ and the characteristic time scale of spin flipping $\tau_{\text {sf }}$, respectively. Note that $\tau$ is much smaller than $\tau_{\mathrm{sf}}[18,25]$, which implies that no-spin-flip collisions happen much faster than spin-flip collisions. Typically, $\tau$ is of $10^{-15} \mathrm{~s}$ (fs) and $\tau_{\mathrm{sf}}$ is of $10^{-12} \mathrm{~s}(\mathrm{ps})$.

Since $\{\hat{\boldsymbol{I}}, \hat{\boldsymbol{\sigma}}\}$ forms a complete set of $2 \times 2$ matrices, one can decompose

$$
W=w I+\hat{\boldsymbol{\sigma}} \cdot \boldsymbol{r},
$$

where $w$ is the spin-independent part and $\boldsymbol{r}$ is the spin-dependent vector. Equation (12) can be separated into two equations, one for $w$

$$
\partial_{t} w+\boldsymbol{v} \cdot \nabla_{\boldsymbol{x}} w-\frac{e}{m} \boldsymbol{E} \cdot \nabla_{\boldsymbol{v}} w=-\frac{w-\bar{w}}{\tau},
$$

and the other for $\boldsymbol{r}$

$$
\begin{aligned}
\partial_{t}(\hat{\boldsymbol{\sigma}} \cdot \boldsymbol{r})+\boldsymbol{v} \cdot \nabla_{\boldsymbol{x}}(\hat{\boldsymbol{\sigma}} \cdot \boldsymbol{r})-\frac{e}{m} \boldsymbol{E} \cdot \nabla_{\boldsymbol{v}}(\hat{\boldsymbol{\sigma}} \cdot \boldsymbol{r})+\frac{J}{\hbar} \hat{\boldsymbol{\sigma}} \cdot(\boldsymbol{m} \times \boldsymbol{r}) \\
=-\frac{(\hat{\boldsymbol{\sigma}} \cdot \boldsymbol{r})-\overline{(\hat{\boldsymbol{\sigma}} \cdot \boldsymbol{r})}}{\tau}-\frac{2}{\tau_{\mathrm{sf}}} \overline{(\hat{\boldsymbol{\sigma}} \cdot \boldsymbol{r})},
\end{aligned}
$$

where we have used the fact that $-\frac{\mathrm{i}}{2 \hbar}[J \hat{\boldsymbol{\sigma}} \cdot \boldsymbol{m}(\boldsymbol{x}, t), W(\boldsymbol{x}, \boldsymbol{v}, t)]=\frac{J}{\hbar} \hat{\boldsymbol{\sigma}} \cdot(\boldsymbol{m} \times \boldsymbol{r})$.

We also notice that (4)-(7), and (14) lead to

$$
\begin{aligned}
& n(\boldsymbol{x}, t)=2 \int_{\mathbb{R}^{3}} w \mathrm{~d} \boldsymbol{v}, \quad \boldsymbol{j}_{n}(\boldsymbol{x}, t)=2 \int_{\mathbb{R}^{3}} w \boldsymbol{v} \mathrm{d} \boldsymbol{v}, \\
& \boldsymbol{s}(\boldsymbol{x}, t)=2 \int_{\mathbb{R}^{3}} \boldsymbol{r} \mathrm{d} \boldsymbol{v}, \quad J_{\boldsymbol{s}}(\boldsymbol{x}, t)=2 \int_{\mathbb{R}^{3}} \boldsymbol{v} \otimes \boldsymbol{r} \mathrm{d} \boldsymbol{v} .
\end{aligned}
$$


Taking the first and second moment of (15) produces

$$
\begin{aligned}
& \partial_{t} n(\boldsymbol{x}, t)+\nabla \cdot \boldsymbol{j}_{n}(\boldsymbol{x}, t)=0 \\
& \partial_{t} \boldsymbol{j}_{n}(\boldsymbol{x}, t)+\int_{\mathbb{R}^{3}}(\boldsymbol{v} \otimes \boldsymbol{v}) \cdot \nabla w(\boldsymbol{x}, \boldsymbol{v}, t) \mathrm{d} \boldsymbol{v}+\frac{e}{m} \boldsymbol{E} n(\boldsymbol{x}, t)=-\frac{\boldsymbol{j}_{n}(\boldsymbol{x}, t)}{\tau} .
\end{aligned}
$$

Taking the first and second moment of (16) produces

$$
\begin{aligned}
& \partial_{t} \boldsymbol{s}(\boldsymbol{x}, t)+\nabla \cdot J_{\boldsymbol{s}}(\boldsymbol{x}, t)+\frac{J}{\hbar} \boldsymbol{m} \times \boldsymbol{s}=-\frac{\boldsymbol{s}(\boldsymbol{x}, t)}{\tau_{\mathrm{sf}}} \\
& \partial_{t} J_{\boldsymbol{s}}(\boldsymbol{x}, t)+\int_{\mathbb{R}^{3}}(\boldsymbol{v} \otimes \boldsymbol{v}) \cdot \nabla \boldsymbol{r}(\boldsymbol{x}, \boldsymbol{v}, t) \mathrm{d} \boldsymbol{v}+\frac{e}{m} \boldsymbol{E} \otimes \boldsymbol{s}(\boldsymbol{x}, t) \\
& \quad+\frac{J}{\hbar} \varepsilon_{j k l} \boldsymbol{m}_{k}\left(J_{\boldsymbol{s}}\right)_{i l}(\boldsymbol{x}, t)=-\frac{J_{\boldsymbol{s}}(\boldsymbol{x}, t)}{\tau} .
\end{aligned}
$$

Note that (19)-(20) and (21)- (22) do not form closed systems for $\left\{n(\boldsymbol{x}, t), \boldsymbol{j}_{n}(\boldsymbol{x}, t)\right\}$ and $\left\{\boldsymbol{s}(\boldsymbol{x}, t), J_{\boldsymbol{s}}(\boldsymbol{x}, t)\right\}$ yet since (20) and (22) depend on the second moment of $w$ and $\boldsymbol{r}$ which in general are not functions of density and current.

There exists a vast literature on closure-related problems for different equations, such as the Boltzmann equation [27], the relaxation-time Wigner equation [1], and the Wigner-Fokker-Plank equation [3, 2]. The BBGKY hierarchy is a standard procedure to derive a system of equations for the moments of the distribution and a truncation is required for higher order moments to close the system. For example, with the Maxwellian velocity distribution as the equilibrium state, one obtains the Euler system by closing the classical Boltzmann equation with a quadratic collisional term [27]; see also related works in $[11,10,7,6,39,20,28]$. However, our problem is like a linear neutron transport equation with a linear collision term with two relaxation time scales $\tau$ and $\tau_{\text {sf }}$, which is then coupled to the magnetization dynamics. Therefore, the classical closures cannot be directly applied. The kinetic equation for chemotaxis is similar to the kinetic model for spin dynamics in the sense that both equations have similar forms with similar linear relaxation collision terms. In the modeling of chemotaxis, the tumbleand-run pattern of $E$. coli also exhibits two time scales, one for tumbling and the other one for running. Therefore, the closure assumption here is motivated by the closure conditions proposed by Hillen for the modeling of chemotaxis to get hyperbolic models and it has been shown to satisfy a minimization principle [23]. We truncate the higher order moments at an approximate level and project them into the subspace spanned by lower order moments.

We project the second moment into the space spanned by the density and current functions, i.e., to approximate $w(\boldsymbol{x}, \boldsymbol{v}, t)$ by a linear function of $\boldsymbol{v}$, (23)

$w(\boldsymbol{x}, \boldsymbol{v}, t)=f(\boldsymbol{v})\left(\gamma_{0} n(\boldsymbol{x}, t)+\gamma_{1} \boldsymbol{m} \cdot \boldsymbol{s}(\boldsymbol{x}, t)+\gamma_{2} \cdot \boldsymbol{v} n_{1}(\boldsymbol{x}, t)+\gamma_{3} \boldsymbol{v} \cdot \boldsymbol{m}_{1}(\boldsymbol{x}, t)\right)$,

where where $f(\boldsymbol{v})$ is a normalized Gaussian function to make $w$ decay at infinity of momentum space. 
By (23), Equation (20) becomes

$$
\begin{aligned}
\partial_{t} \boldsymbol{j}_{n}(\boldsymbol{x}, t)+\gamma_{0} \overline{v^{2}} \nabla_{\boldsymbol{x}} n(\boldsymbol{x}, t)+\gamma_{1} \overline{v^{2}} \nabla_{\boldsymbol{x}} \boldsymbol{s}(\boldsymbol{x}, t) \boldsymbol{m} & \\
& +\frac{e}{m} \boldsymbol{E} n(\boldsymbol{x}, t)=-\frac{\boldsymbol{j}_{n}(\boldsymbol{x}, t)}{\tau},
\end{aligned}
$$

where $\overline{v^{2}}=\frac{1}{4 \pi} \int|\boldsymbol{v}|^{2} \mathrm{~d} \Omega \boldsymbol{v}$

Similarly, we can assume

$\boldsymbol{r}(\boldsymbol{x}, \boldsymbol{v}, t)=g(\boldsymbol{v})\left(\gamma_{0}^{\prime} \boldsymbol{m} n(\boldsymbol{x}, t)+\gamma_{1}^{\prime} \boldsymbol{s}(\boldsymbol{x}, t)+\gamma_{2}^{\prime} \boldsymbol{v} n_{1}(\boldsymbol{x}, t)+\gamma_{3}^{\prime} \boldsymbol{v}\left(\boldsymbol{m} \cdot \boldsymbol{m}_{1}(\boldsymbol{x}, t)\right)\right)$,

where $g(\boldsymbol{v})$ is a normalized Gaussian function to make $\boldsymbol{r}$ decay at infinity of momentum space. Equation (22) then becomes

$$
\begin{aligned}
\partial_{t} J_{\boldsymbol{s}}(\boldsymbol{x}, t)+\gamma_{0}^{\prime} \overline{v^{2}} \nabla_{\boldsymbol{x}} n(\boldsymbol{x}, t) \otimes \boldsymbol{m}+\gamma_{1}^{\prime} \overline{v^{2}} \nabla_{\boldsymbol{x}} \boldsymbol{s}(x, t) \\
\quad+\frac{e}{m} \boldsymbol{E} \otimes \boldsymbol{s}(\boldsymbol{x}, t)+\frac{J}{\hbar} \varepsilon_{j k l} \boldsymbol{m}_{k}\left(J_{\boldsymbol{s}}\right)_{i l}(\boldsymbol{x}, t)=-\frac{J_{\boldsymbol{s}}(\boldsymbol{x}, t)}{\tau} .
\end{aligned}
$$

Note that the last term on the left-hand-side of (25) is independent of the closure strategy we use.

In summary, we obtain a closed moment system for $\left\{n(\boldsymbol{x}, t), \boldsymbol{j}_{n}(\boldsymbol{x}, t), \boldsymbol{s}(\boldsymbol{x}, t), J_{\boldsymbol{s}}(\boldsymbol{x}, t)\right\}$, which provides a mean-field description for the spin dynamics,

$$
\begin{aligned}
\partial_{t} n(\boldsymbol{x}, t) & +\nabla_{\boldsymbol{x}} \boldsymbol{j}_{n}(\boldsymbol{x}, t)=0 \\
\partial_{t} \boldsymbol{j}_{n}(\boldsymbol{x}, t) & +\gamma_{0} \overline{v^{2}} \nabla_{\boldsymbol{x}} n(\boldsymbol{x}, t)+\gamma_{1} \overline{v^{2}} \nabla_{\boldsymbol{x}} \boldsymbol{s}(\boldsymbol{x}, t) \boldsymbol{m} \\
& +\frac{e}{m} \boldsymbol{E} n(\boldsymbol{x}, t)=-\frac{\boldsymbol{j}_{n}(\boldsymbol{x}, t)}{\tau}, \\
\partial_{t} \boldsymbol{s}(\boldsymbol{x}, t) & +\nabla_{\boldsymbol{x}} J_{\boldsymbol{s}}(\boldsymbol{x}, t)+\frac{J}{\hbar} \boldsymbol{m} \times \boldsymbol{s}(\boldsymbol{x}, t)=-\frac{\boldsymbol{s}(\boldsymbol{x}, t)}{\tau_{\mathrm{sf}}} \\
\partial_{t} J_{\boldsymbol{s}}(\boldsymbol{x}, t) & +\gamma_{0}^{\prime} \overline{v^{2}} \nabla_{\boldsymbol{x}} n(\boldsymbol{x}, t) \otimes \boldsymbol{m}+\gamma_{1}^{\prime} \overline{v^{2}} \nabla_{\boldsymbol{x}} \boldsymbol{s}(\boldsymbol{x}, t) \\
& +\frac{e}{m} \boldsymbol{E} \otimes \boldsymbol{s}(\boldsymbol{x}, t)+\frac{J}{\hbar} \varepsilon_{j k l} \boldsymbol{m}_{k}\left(J_{\boldsymbol{s}}\right)_{i l}(\boldsymbol{x}, t)=-\frac{J_{\boldsymbol{s}}(\boldsymbol{x}, t)}{\tau} .
\end{aligned}
$$

2.1.3. Comparison to the linear response theory in [42]. Quasi-static approximation of $\boldsymbol{j}_{n}(\boldsymbol{x}, t)$ in (27) produces

$$
\boldsymbol{j}_{n}(\boldsymbol{x}, t)=-\frac{e}{m} \tau \boldsymbol{E} n(\boldsymbol{x}, t)-\gamma_{0} \overline{v^{2}} \tau \nabla_{\boldsymbol{x}} n(\boldsymbol{x}, t)-\gamma_{1} \overline{v^{2}} \tau \nabla_{\boldsymbol{x}} \boldsymbol{s}(\boldsymbol{x}, t) \boldsymbol{m} .
$$

Similarly, quasi-static approximation of $J_{\boldsymbol{s}}(\boldsymbol{x}, t)$ in $(29)$ yields

$$
J_{\boldsymbol{s}}(\boldsymbol{x}, t) \boldsymbol{A}(\boldsymbol{m})=-\frac{e}{m} \tau \boldsymbol{E} \otimes \boldsymbol{s}(\boldsymbol{x}, t)-\gamma_{0}^{\prime} \overline{v^{2}} \tau \nabla_{\boldsymbol{x}} n(\boldsymbol{x}, t) \otimes \boldsymbol{m}-\gamma_{1}^{\prime} \overline{v^{2}} \tau \nabla_{\boldsymbol{x}} \boldsymbol{s}(\boldsymbol{x}, t),
$$

where

$$
\boldsymbol{A}(\boldsymbol{m})=\left(\begin{array}{ccc}
1 & \frac{J \tau}{\hbar} m_{3} & -\frac{J \tau}{\hbar} m_{2} \\
-\frac{J \tau}{\hbar} m_{3} & 1 & \frac{J \tau}{\hbar} m_{1} \\
\frac{J \tau}{\hbar} m_{2} & -\frac{J \tau}{\hbar} m_{1} & 1
\end{array}\right)
$$


The governing equations for $n$ and $s$ then become

$$
\begin{aligned}
\partial_{t} n(\boldsymbol{x}, t) & +\nabla_{\boldsymbol{x}} \boldsymbol{j}_{n}(\boldsymbol{x}, t)=0 \\
\boldsymbol{j}_{n}(\boldsymbol{x}, t) & =-\frac{e}{m} \tau \boldsymbol{E} n(\boldsymbol{x}, t)-\gamma_{0} \overline{v^{2}} \tau \nabla_{\boldsymbol{x}} n(\boldsymbol{x}, t)-\gamma_{1} \overline{v^{2}} \tau \nabla_{\boldsymbol{x}} \boldsymbol{s}(\boldsymbol{x}, t) \boldsymbol{m}, \\
\partial_{t} \boldsymbol{s}(\boldsymbol{x}, t) & +\nabla_{\boldsymbol{x}} J_{\boldsymbol{s}}(\boldsymbol{x}, t)+\frac{J}{\hbar} \boldsymbol{m} \times \boldsymbol{s}(\boldsymbol{x}, t)=-\frac{\boldsymbol{s}(\boldsymbol{x}, t)}{\tau_{\mathrm{sf}}}, \\
J_{\boldsymbol{s}}(\boldsymbol{x}, t) \boldsymbol{A}(\boldsymbol{m}) & =-\frac{e}{m} \tau \boldsymbol{E} \otimes \boldsymbol{s}(x, t)-\gamma_{0}^{\prime} \overline{v^{2}} \tau \nabla_{\boldsymbol{x}} n(\boldsymbol{x}, t) \otimes \boldsymbol{m}-\gamma_{1}^{\prime} \overline{v^{2}} \tau \nabla_{\boldsymbol{x}} \boldsymbol{s}(\boldsymbol{x}, t) .
\end{aligned}
$$

For the characteristic parameters of Permalloy [37], we obtain the averaged velocity $\bar{v} \sim 5 \times 10^{6} \mathrm{~m} / \mathrm{s}$, and $\tau \sim 10^{-15}$ s. The quasi-static approximation corresponds to the parabolic scaling of (27) and (29), i.e., the long time behavior of the system is dominated by relaxation. The equations above are only valid in the diffusive regime. The moment system (26)-(29), however, is valid not just in the diffusive regime, but also in other regimes, such as the hyperbolic regime. This becomes important if fast spin dynamics is present [30].

In [42], the authors derived a diffusion model for spin dynamics based on the linear response theory. The constitutive relations they have are

$$
\begin{aligned}
\boldsymbol{j}_{n} & =2 C_{0} \boldsymbol{E}-2 \beta^{\prime} D_{0} \nabla_{\boldsymbol{x}} \boldsymbol{s} \boldsymbol{m}, \\
J_{\boldsymbol{s}} & =2 \beta C_{0} \boldsymbol{E} \otimes \boldsymbol{m}-2 D_{0} \nabla_{\boldsymbol{x}} \boldsymbol{s}
\end{aligned}
$$

under the assumption that $n(\boldsymbol{x}, t)$ is homogeneous in space and time and $\boldsymbol{s}$ aligns up with $\boldsymbol{m}$. Here $C_{0}$ is the conductivity, $D_{0}$ is the diffusivity, $\beta$ is the spin polarization parameter for conductivity, and $\beta^{\prime}$ is the other spin polarization parameter for diffusivity. The diffusion equation for spin dynamics is

$$
\partial_{t} \boldsymbol{s}(\boldsymbol{x}, t)=-\nabla_{\boldsymbol{x}} J_{\boldsymbol{s}}(\boldsymbol{x}, t)-2 D_{0}(\boldsymbol{x}) \frac{\boldsymbol{s}(\boldsymbol{x}, t)}{\lambda_{\mathrm{sf}}^{2}}-2 D_{0}(\boldsymbol{x}) \frac{\boldsymbol{s}(\boldsymbol{x}, t) \times \boldsymbol{m}}{\lambda_{J}^{2}}
$$

with

$$
J_{\boldsymbol{s}}(\boldsymbol{x}, t)=\frac{\beta \mu_{\mathrm{B}}}{e} \boldsymbol{j}_{n} \otimes \boldsymbol{m}-2 D_{0}(\boldsymbol{x})\left[\nabla \boldsymbol{s}-\beta \beta^{\prime}(\nabla \boldsymbol{s} \cdot \boldsymbol{m}) \otimes \boldsymbol{m}\right],
$$

which is obtained by solving $\boldsymbol{E}$ in terms of $\boldsymbol{j}_{n}$ in (32) first and then substituting it into (33).

Under the same assumption in [42], (30) and (31) become

$$
\begin{array}{r}
\boldsymbol{j}_{n}=2 C_{0} \tau \boldsymbol{E}-2 \beta^{\prime} D_{0}(x) \nabla_{\boldsymbol{x}} \boldsymbol{s} \boldsymbol{m}, \\
J_{\boldsymbol{s}} \boldsymbol{A}(\boldsymbol{m})=2 \beta C_{0} \boldsymbol{E} \otimes \boldsymbol{m}-2 D_{0}(x) \nabla_{\boldsymbol{x}} \boldsymbol{s},
\end{array}
$$

where $2 C_{0}=-\frac{e}{m} n(\boldsymbol{x}, t), 2 \beta^{\prime} D_{0}(x)=\gamma_{1} \overline{v^{2}} \tau, 2 \beta C_{0} \boldsymbol{m}=-\frac{e}{m} \tau \boldsymbol{s}$, and $2 D_{0}(x)=$ $\gamma_{1}^{\prime} \overline{v^{2}} \tau$. Note that the factor 2 comes from the fact that we include 2 in (17) and (18), while 2 is kept explicitly in [42]; see (5) and (6) in [42]. Playing 
with the same trick to obtain (35), we get

$$
J_{\boldsymbol{s}}(\boldsymbol{x}, t) \boldsymbol{A}(\boldsymbol{m})=\frac{\beta \mu_{\mathrm{B}}}{e} \boldsymbol{j}_{n} \otimes \boldsymbol{m}-2 D_{0}(\boldsymbol{x})\left[\nabla \boldsymbol{s}-\beta \beta^{\prime}(\nabla \boldsymbol{s} \cdot \boldsymbol{m}) \otimes \boldsymbol{m}\right]
$$

and

$$
\boldsymbol{A}(\boldsymbol{m})=\left(\begin{array}{ccc}
1 & \frac{\tau}{\tau_{J}} m_{3} & -\frac{\tau}{\tau_{J}} m_{2} \\
-\frac{\tau}{\tau_{J}} m_{3} & 1 & \frac{\tau}{\tau_{J}} m_{1} \\
\frac{\tau}{\tau_{J}} m_{2} & -\frac{\tau}{\tau_{J}} m_{1} & 1
\end{array}\right),
$$

where $\tau_{J}=\hbar / J$ is the characteristic time scale of the spin-magnetization coupling.

In the diffusive regime, the moment system becomes

$$
\partial_{t} \boldsymbol{s}(\boldsymbol{x}, t)=-\nabla_{\boldsymbol{x}} J_{\boldsymbol{s}}(\boldsymbol{x}, t)-2 D_{0}(\boldsymbol{x}) \frac{\boldsymbol{s}(\boldsymbol{x}, t)}{\lambda_{\mathrm{sf}}^{2}}-2 D_{0}(\boldsymbol{x}) \frac{\boldsymbol{s}(\boldsymbol{x}, t) \times \boldsymbol{m}}{\lambda_{J}^{2}},
$$

which is the same as (34) except that $J_{\boldsymbol{s}}$ satisfies (36).

As $\tau_{J} \gg \tau, A(\boldsymbol{m})$ approaches $\mathrm{I}$, the moment system in the diffusive regime recovers the diffusion model derived from the linear response theory [42], which corresponds to the limit of weak spin-magnetization coupling. However, beyond that, these two models are different. In general, $A(\boldsymbol{m})$ has eigenvalues 1, $1 \pm \frac{\tau}{\tau_{J}} i$ and $\operatorname{det} \boldsymbol{A}(\boldsymbol{m})=1+\left(\frac{\tau}{\tau_{J}}\right)^{2}$. Since $\tau$ is the characteristic time of the electron collision and $\lambda=\sqrt{2 D_{0} \tau}$, we have

$$
\frac{\tau}{\tau_{J}}=\frac{\lambda_{J}^{2}}{\lambda^{2}}
$$

where $\lambda$ is the electron mean free path due to the electron collision. Physical interpretation of this model will be given in a subsequent publication [12]. For a permalloy, $\lambda=4 \mathrm{~nm}$. Values of $\tau_{J}$ and $\lambda_{J}=\sqrt{2 D_{0} \tau_{J}}$ depend on the strength of spin-magnetization coupling, which will be tested in section 4 . For completeness, we set

$$
\begin{aligned}
\boldsymbol{s}(\boldsymbol{x}, 0) & =\mathbf{0}, \forall \boldsymbol{x} \in \Omega, \\
\frac{\partial \boldsymbol{s}}{\partial \nu} & =\mathbf{0}, \text { on } \partial \Omega
\end{aligned}
$$

with $\nu$ the outward unit normal vector on $\partial \Omega$.

2.2. Magnetization dynamics. The dynamics of the magnetization, in the presence of a spin-transfer torque, is described by the LLG equation $([26,19])$,

$$
\frac{\partial \boldsymbol{m}}{\partial t}=-\gamma \boldsymbol{m} \times\left(\boldsymbol{H}_{\mathrm{e}}+J \boldsymbol{s}\right)+\alpha \boldsymbol{m} \times \frac{\partial \boldsymbol{m}}{\partial t},
$$

where the magnetization $\boldsymbol{m}(\boldsymbol{x}, t)$, normalized to $|\boldsymbol{m}|=1$, is defined over $\Sigma$, and the spin $\boldsymbol{s}(\boldsymbol{x}, t)$ is defined over $\Omega$. Here $\gamma=1.76 \times 10^{11}(\mathrm{Ts})^{-1}$ is the gyromagnetic ratio, $J$ is the coupling strength between the spin and the magnetization, and $\alpha$ is the dimensionless damping constant, which we take to be 0.1 here. The first term on the right-hand-side describes the 
precession of the magnetization around the local effective field $\boldsymbol{H}_{\mathrm{e}}$ plus spin contribution $J s$, while the second term is known as the Gilbert damping. Neumann boundary conditions are used for (41)

$$
\frac{\partial \boldsymbol{m}}{\partial \nu}=0, \quad \text { on } \partial \Sigma,
$$

where $\nu$ is the outward unit normal vector on $\partial \Sigma$.

The effective field $\boldsymbol{H}_{\mathrm{e}}$ has the form

$$
\boldsymbol{H}_{\mathrm{e}}=-\frac{2 K_{\mathrm{u}}}{M_{\mathrm{S}}}\left(m_{2} \boldsymbol{e}_{2}+m_{3} \boldsymbol{e}_{3}\right)+\frac{2 C_{\mathrm{ex}}}{M_{\mathrm{S}}} \Delta \boldsymbol{m}+\mu_{0}\left(\boldsymbol{H}_{\mathrm{s}}+\boldsymbol{H}_{0}\right),
$$

and can be calculated as $-\frac{\delta F_{\mathrm{LL}}}{\delta \boldsymbol{m}}$, where $F_{\mathrm{LL}}$ is the Landau-Lifshitz energy given by

$$
F_{\mathrm{LL}}=\frac{K_{\mathrm{u}}}{M_{\mathrm{S}}} \int_{\Sigma}\left(m_{2}^{2}+m_{3}^{2}\right)+\frac{C_{\mathrm{ex}}}{M_{\mathrm{S}}} \int_{\Sigma}|\nabla \boldsymbol{m}|^{2}-\frac{\mu_{0}}{2} \int_{\Sigma} \boldsymbol{H}_{\mathrm{s}} \cdot \boldsymbol{m}-\mu_{0} \int_{\Sigma} \boldsymbol{H}_{0} \cdot \boldsymbol{m} \boldsymbol{.}
$$

In $(44), \boldsymbol{e}_{2}=(0,1,0), \boldsymbol{e}_{3}=(0,0,1)$, and $\mu_{0}=4 \pi \times 10^{-7} \mathrm{~N} / \mathrm{A}^{2}$ is the permeability of vacuum. $K_{\mathrm{u}}$ and $C_{\mathrm{ex}}$ are materials constants, and $M_{\mathrm{s}}$ is the saturation magnetization which is also material-dependent. For physical constants characteristic of the permalloy, $K_{\mathrm{u}}=5.0 \times 10^{2} \mathrm{~J} / \mathrm{m}^{3}, C_{\mathrm{ex}}=1.3 \times 10^{-11} \mathrm{~J} / \mathrm{m}$, and $M_{\mathrm{s}}=8.0 \times 10^{5} \mathrm{~A} / \mathrm{m} . \boldsymbol{H}_{0}$ is the externally applied magnetic field and $\boldsymbol{H}_{\mathrm{S}}$ is the stray field, given by $\boldsymbol{H}_{\mathrm{S}}=-\nabla u$, where $u$ satisfies the following magnetostatic equation

$$
\begin{aligned}
\Delta u & =\operatorname{div} \boldsymbol{m}, \quad \boldsymbol{x} \in \Sigma \\
\Delta u & =0, \quad \boldsymbol{x} \in \bar{\Sigma}^{c}
\end{aligned}
$$

with jump boundary conditions

$$
\begin{aligned}
{[u]_{\partial \Sigma} } & =0, \\
{\left[\frac{\partial u}{\partial \nu}\right]_{\partial \Sigma} } & =-\boldsymbol{m} \cdot \nu,
\end{aligned}
$$

where $[\cdot]$ represents the jump at $\partial \Sigma$. The solution to this equation is

$$
u(\boldsymbol{x})=\int_{\Sigma} \nabla N(\boldsymbol{x}-\boldsymbol{y}) \cdot \boldsymbol{m}(\boldsymbol{y}) \mathrm{d} \boldsymbol{y},
$$

where $N(\boldsymbol{x})=-1 /(4 \pi|\boldsymbol{x}|)$ is the Newtonian potential.

\section{Numerical method}

We describe in Figure 1 a standard device for ferromagnetic multilayers, where two ferromagnetic layers (FM1 and FM2) are separated by a nonmagnetic metallic spacer (NM). We consider an electron current applied perpendicular to the device. Heuristically, the electrons in the current are polarized in the first layer, and exert an additional torque on the magnetization in the second layer. 
3.1. Spatial discretization. The domain is discretized using a uniform mesh. The magnetization and the spin accumulation are defined at the center of the computational cells. Spatial derivatives are approximated using standard centered differences away from the interface between ferromagnetic and nonmagnetic layers, and using one-sided differences near the interface $[17]$.

3.2. Temporal discretization. Although explicit schemes may achieve high order of accuracy both in space and time, the time step size is severely constrained by the stability of the numerical scheme. Considering that the precession timescale is of the order of a picosecond, numerical stability issues can have a significant effect on the performance of the algorithm. In order to overcome the stability constraint of explicit schemes, one usually resorts to implicit schemes. However, due to the strong nonlinearities present in both the gyromagnetic and damping terms in the LLG equation, a direct implicit discretization of the system is not efficient and is difficult to implement.

Here we use the Gauss-Seidel Projection Method (GSPM) introduced in [41] to solve (41). The GSPM is a semi-implicit scheme that requires only the solution of linear systems of equations; the nonlinearity in the equations is introduced a posteriori, resulting in an unconditionally stable method. For the coupled spin-magnetization system (41)-(34), a splitting method for (34) is proposed, which together with GSPM for (41) provides an unconditionally stable scheme for the coupled system.

Note that the spectral splitting method (SSM) proposed in [17] can not be directly applied for the computation of (38) due to the existence of $\boldsymbol{A}(\boldsymbol{m})$ which introduces the inhomogeneous diffusion. On the other hand, explicit solvers will significantly increase the computational cost since the time step is limited by the diffusion. Therefore, we propose to use a homogeneous diffusion as the penalization, which can be dealt with easily by SSM in [17], and the difference between the homogeneous and inhomogeneous diffusion is less stiff, and thus can be treated explicitly. The details of the algorithm are given in (54) and (55). This is motivated by the penalization techniques used for variable coefficient diffusion equations [14], phase field models [4] and the Boltzmann equation [16].

This splitting procedure is then coupled to GSPM for magnetization dynamics (41). Stability of the splitting method is confirmed by numerical examples.

For completeness, we include here a description of the GSPM, when spin currents are present. It is convenient to rewrite equation (41) in the following form:

$$
\frac{\partial \boldsymbol{m}}{\partial t}=-\boldsymbol{m} \times(\epsilon \Delta \boldsymbol{m}+\boldsymbol{f}(\boldsymbol{m}, \boldsymbol{s}))-\alpha \boldsymbol{m} \times(\boldsymbol{m} \times(\epsilon \Delta \boldsymbol{m}+\boldsymbol{f}(\boldsymbol{m}, \boldsymbol{s}))),
$$

where $\epsilon=2 C_{\text {ex }} \gamma /\left(M_{\mathrm{s}}\left(1+\alpha^{2}\right)\right)$, and

$$
\boldsymbol{f}(\boldsymbol{m}, \boldsymbol{s})=\frac{\gamma}{1+\alpha^{2}}\left(-\frac{2 K_{\mathrm{u}}}{M_{\mathrm{s}}}\left(m_{2} \boldsymbol{e}_{2}+m_{3} \boldsymbol{e}_{3}\right)+\mu_{0}\left(\boldsymbol{H}_{\mathrm{s}}+\boldsymbol{H}_{0}\right)+J \boldsymbol{s}\right) .
$$


Equation (48) is obtained from (41) by evaluating the vector product of $\boldsymbol{m}$ and (41), and solving for $\boldsymbol{m} \times \frac{\partial \boldsymbol{m}}{\partial t}$.

Given $\boldsymbol{m}^{n}=\boldsymbol{m}\left(t_{n}\right)$ and $\boldsymbol{s}^{n}=\boldsymbol{s}\left(t_{n}\right)$, in the GSPM we solve (48) in three steps:

Step 1: Implicit Gauss-Seidel. Define

$$
\begin{aligned}
g_{i}^{n} & =\left(I-\epsilon \Delta t \Delta_{h}\right)^{-1}\left(m_{i}^{n}+\Delta t f_{i}^{n}\right), \\
g_{i}^{*} & =\left(I-\epsilon \Delta t \Delta_{h}\right)^{-1}\left(m_{i}^{*}+\Delta t f_{i}^{*}\right), \quad i=1,2,3
\end{aligned}
$$

and

$$
\left(\begin{array}{c}
m_{1}^{*} \\
m_{2}^{*} \\
m_{3}^{*}
\end{array}\right)=\left(\begin{array}{c}
m_{1}^{n}+\left(g_{2}^{n} m_{3}^{n}-g_{3}^{n} m_{2}^{n}\right) \\
m_{2}^{n}+\left(g_{3}^{n} m_{1}^{*}-g_{1}^{*} m_{3}^{n}\right) \\
m_{3}^{n}+\left(g_{1}^{*} m_{2}^{*}-g_{2}^{*} m_{1}^{*}\right)
\end{array}\right)
$$

where $f_{i}^{n}=f_{i}\left(\boldsymbol{m}^{n}, \boldsymbol{s}^{n}\right)$, and $f_{i}^{*}=f_{i}\left(\boldsymbol{m}^{*}, \boldsymbol{s}^{n}\right)$, i.e., the most current values for $\boldsymbol{m}$ are used in $\boldsymbol{f}^{*}$. Note that the value of $\boldsymbol{s}$ is frozen at $t=t_{n}$.

Step 2: Heat flow without constraints. Solve the Backward-Euler-type equations

$$
\left(\begin{array}{c}
m_{1}^{* *} \\
m_{2}^{* *} \\
m_{3}^{* *}
\end{array}\right)=\left(\begin{array}{c}
m_{1}^{*}+\alpha \Delta t\left(\epsilon \Delta_{h} m_{1}^{* *}+f_{1}^{*}\right) \\
m_{2}^{*}+\alpha \Delta t\left(\epsilon \Delta_{h} m_{2}^{* *}+f_{2}^{*}\right) \\
m_{3}^{*}+\alpha \Delta t\left(\epsilon \Delta_{h} m_{3}^{* *}+f_{3}^{*}\right)
\end{array}\right)
$$

Step 3: Projection onto $S^{2}$, in order to normalize the magnetization.

$$
\left(\begin{array}{c}
m_{1}^{n+1} \\
m_{2}^{n+1} \\
m_{3}^{n+1}
\end{array}\right)=\frac{1}{\left|m^{* *}\right|}\left(\begin{array}{c}
m_{1}^{* *} \\
m_{2}^{* *} \\
m_{3}^{* *}
\end{array}\right)
$$

Only linear systems of equations are solved, and therefore the complexity of the splitting algorithm is comparable to that of solving the linear heat equation using the Backward Euler method.

Following [17], we solve the spin diffusion equation (38) coupled to the LLG (48) in three steps:

Step 1: Solve the Cauchy problem

$$
\begin{aligned}
\frac{d \widetilde{\boldsymbol{s}}}{\mathrm{d} t} & =-2 D_{0}(\boldsymbol{x}) \frac{\widetilde{\boldsymbol{s}}}{\lambda_{\mathrm{sf}}^{2}}-2 D_{0}(\boldsymbol{x}) \frac{\widetilde{\boldsymbol{s}} \times \boldsymbol{m}^{n}}{\lambda_{J}^{2}}, \\
\widetilde{\boldsymbol{s}}\left(t_{n}\right) & =\boldsymbol{s}^{n} .
\end{aligned}
$$

The solution to this problem can be found analytically. 
Step 2: Solve the following system of equations:

$$
\begin{aligned}
\frac{\boldsymbol{s}^{n+1}-\widetilde{\boldsymbol{s}}\left(t_{n+1}\right)}{\Delta t} & =\nabla_{h} \cdot\left(2 D_{0}(\boldsymbol{x}) \nabla_{h} \boldsymbol{s}^{n+1} \boldsymbol{I}\right) \\
& +\nabla_{h} \cdot\left(\left(2 D_{0}(\boldsymbol{x}) \nabla_{h} \widetilde{\boldsymbol{s}}\left(t_{n+1}\right)\left(\boldsymbol{A}^{-1}\left(\boldsymbol{m}^{n}\right)-\boldsymbol{I}\right)\right)\right. \\
& -\nabla_{h}\left(\left(\beta \beta^{\prime} 2 D_{0}(\boldsymbol{x})\left(\left(\boldsymbol{m}^{n}, \nabla_{h} \boldsymbol{s}^{n}\right) \boldsymbol{m}^{n}\right)\right.\right. \\
& \left.\left.+\frac{\beta \mu_{\mathrm{B}}}{e}\left(\boldsymbol{j}_{n} \otimes \boldsymbol{m}^{n}\right)\right) \boldsymbol{A}^{-1}\left(\boldsymbol{m}^{n}\right)\right) .
\end{aligned}
$$

Due to material heterogeneity in the $z$ direction, FFTW is used only in the $x$ and $y$ directions and the resulting system is therefore heptadiagonal, which can be effectively solved using Gaussian elimination. Note that (55) reduces to (26) in [17] if $\boldsymbol{A}(\boldsymbol{m})=\boldsymbol{I}$.

Step 3: Solve (48) by the GSPM described above.

The splitting method here is first-order accurate in time. In real applications, we usually choose $\Delta t=10^{-13} \mathrm{~s}$, which is at the sub time scale of spin flipping $\tau_{\text {sf }}$, and $h=2 \mathrm{~nm}$ to resolve domain walls and vortices in our ferromagnetic samples. It is worth mentioning that the temporal step size and spatial grid size are comparable after nondimensionalization, which balances the spatial and temporal accuracies.

\section{Results}

We examine the magnetization switching for (41) (38) with different $\tau / \tau_{J}$ values under two different conditions: a) an applied current; b) an applied current and an external magnetic field. Note that if $\tau / \tau_{J}=0$ in (37), (38) recovers (34). Typical values for the remaining parameters are [37]: $D_{0}=10^{-3} \mathrm{~m}^{2} / \mathrm{s}$ for the magnetic layer, and $D_{0}=5 \times 10^{-3} \mathrm{~m}^{2} / \mathrm{s}$ for the nonmagnetic layer, $\lambda_{\mathrm{sf}}=10 \mathrm{~nm}, J=0.1 \mathrm{eV}, \beta=0.9, \beta^{\prime}=0.8$. The simulations presented here were carried out using $h=2 \mathrm{~nm}$, and $\Delta t=10^{-13} \mathrm{~s}$. No appreciable differences were found when smaller values of $h$ or $\Delta t$ were used.

4.1. Current-driven magnetization reversal. One of the main technological applications of spin-polarized transport is the magnetization reversal in a multilayer in the absence of externally applied magnetic fields, as this can allow an increase in the density of magnetic memories. Consider a multilayer with in-plane dimensions $128 \mathrm{~nm} \times 64 \mathrm{~nm}$; see Figure 1 . Choose $D_{1}=200 \mathrm{~nm}, D_{2}=20 \mathrm{~nm}$, and $D_{3}=60 \mathrm{~nm}$. The multilayer is initialized in a uniform state, and it is allowed to reach steady state. Subsequently, a perpendicular current $\boldsymbol{j}_{n}$ is applied for 10 nanoseconds, and then it is removed. 


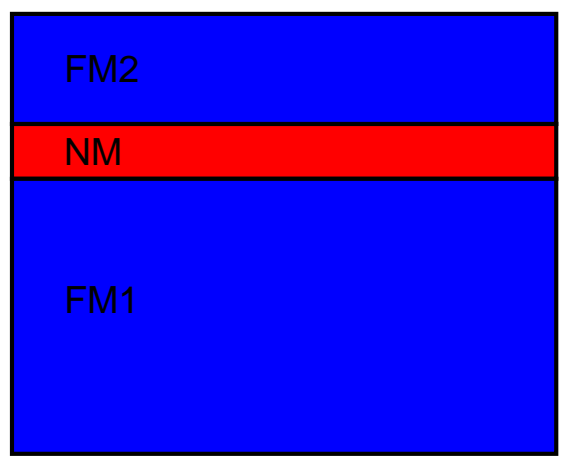

FiguRE 1. A typical ferromagnetic multilayer device: Two ferromagnetic layers (FM1 and FM2) of thickness $D_{1}$ and $D_{3}$, respectively, sandwiched by a nonmagnetic metallic spacer (NM) of thickness $D_{2}$.

Figure 2 shows a $\mathrm{S}$ state, and an intermediate vortex state that nucleates inside the sample during the reversal of the S state. For clarity of presentation, we plot only the in-plane components of the magnetization, measured at the center slice of the top layer.

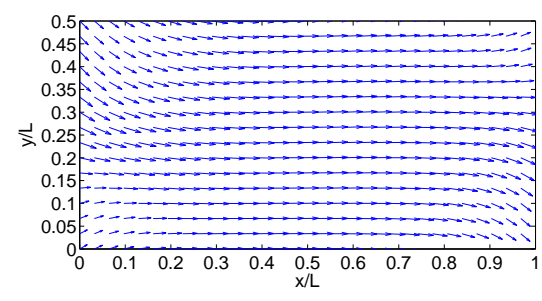

(a) S state

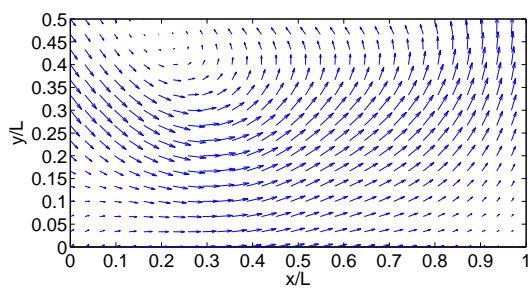

(b) Vortex state

FIgURE 2. States during the magnetization switching: (a): S state; (b): Vortex state.

The average magnetization in the top and bottom layers are plotted as a function of time in Figure 3. For an appropriate current in (38), the magnetization in the top layer was reversed as a consequence of the spincurrents, in agreement with recent experiments.

As the applied current raises gradually, the magnetization of the top layer experiences a transition from the $\mathrm{S}$ state to the vortex state, the reversed $\mathrm{S}$ state, and the vortex state again, respectively. Critical currents for these transitions are recorded in Table 1. It is clear that (38) has a larger effective window for the $\mathrm{S} \rightarrow \mathrm{S}$ transition compared with (34). Since eigenvalues of 


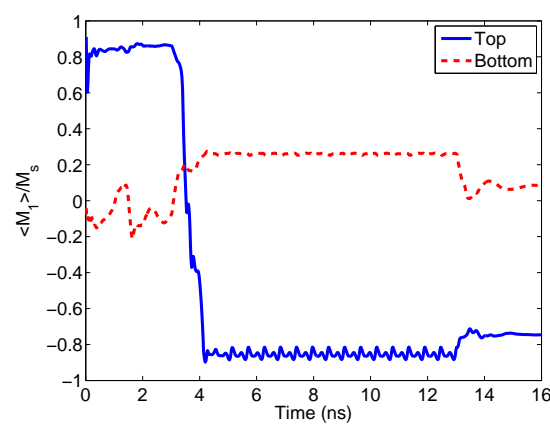

(a) $\boldsymbol{j}_{n}=10.3$

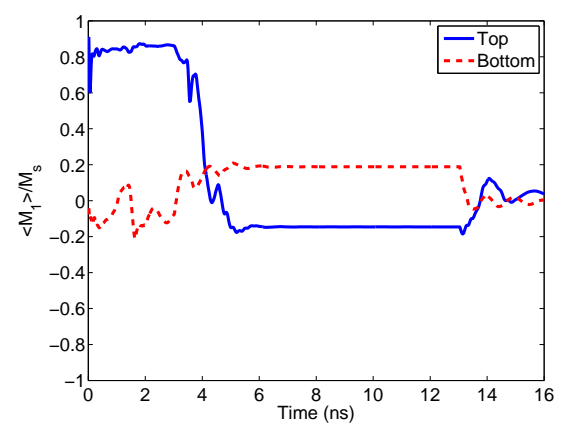

(b) $\boldsymbol{j}_{n}=9.9$

FigURE 3. Results of (41) (38) for a perpendicular current $j_{n}$ with different magnitudes when $\tau / \tau_{J}=1$. (a): $\boldsymbol{j}_{n}=10.3 \times$ $10^{10} \mathrm{~A} / \mathrm{m}^{2} ;(\mathrm{b}): \boldsymbol{j}_{n}=9.9 \times 10^{10} \mathrm{~A} / \mathrm{m}^{2}$.

$\boldsymbol{A}(\boldsymbol{m})$ are always greater than or equal to $1, \boldsymbol{A}(\boldsymbol{m})$ plays an role as a contraction matrix, so critical currents required in (38) are always larger than those required in (34). Table 2 further confirms the above observation. Besides, critical currents increase as the top layer becomes thinner. A careful examination shows that magnetization of the top layer becomes more uniaxial as its thickness reduces, and magnetization switching happens mainly by in-plane rotation with less out-of-plane rotation.

\begin{tabular}{ccccc}
\hline$\tau / \tau_{J}$ & $\mathrm{~S}$ & $\mathrm{~S} \rightarrow$ Vortex & $\mathrm{S} \rightarrow \mathrm{S}$ & $\mathrm{S} \rightarrow$ Vortex \\
\hline 0 & $\leq 7.8$ & {$[7.9,9.8]$} & $\{9.9\}$ & $\geq 10.0$ \\
\hline 1 & $\leq 9.7$ & {$[9.8,10.1]$} & {$[10.2,10.4]$} & $\geq 10.5$ \\
\hline 2 & $\leq 9.9$ & {$[10.0,12.8]$} & {$[12.9,18.5]$} & $\geq 18.6$ \\
\hline
\end{tabular}

TABLE 1. Critical currents $\boldsymbol{j}_{n}$ of magnetization switching for different $\tau / \tau_{J}$ values (unit: $10^{10} \mathrm{~A} / \mathrm{m}^{2}$ ).

\begin{tabular}{ccc}
\hline Thickness $(\mathrm{nm})$ & $\tau / \tau_{J}=0$ & $\tau / \tau_{J}=1$ \\
\hline 60 & $\{9.9\}$ & {$[10.2,10.4]$} \\
\hline 56 & {$[10.5,10.6]$} & {$[12.0,12.2]$} \\
\hline 52 & {$[10.8,11.4]$} & {$[12.7,15.0]$} \\
\hline 48 & {$[12.5,13.1]$} & {$[12.7,17.1]$}
\end{tabular}

TABLE 2. Effective switching window ( $\rightarrow \mathrm{S}$ transition) for different $\tau / \tau_{J}$ values as a function of thickness of the top layer.

4.2. Reduction in the coercive field. Maximal GMR can be achieved if the magnetization of both layers changes from parallel (antiparallel) state to antiparallel (parallel) state. Therefore, understanding the reversal process is of technological importance. 
Consider a multilayer of in-plane dimensions $128 \mathrm{~nm} \times 64 \mathrm{~nm}$, and thicknesses $D_{1}=60 \mathrm{~nm}, D_{2}=10 \mathrm{~nm}$, and $D_{3}=20 \mathrm{~nm}$. We compute the hysteresis loop with and without spin currents. The hysteresis loop is computed in the following way: Initially, an external field of magnitude $\boldsymbol{H}_{0}$ is applied in order to saturate the sample, and the magnetization is allowed to reach an equilibrium state. Once steady state is reached, the applied field is reduced, and the magnetization is allowed to reach equilibrium again. This process is repeated, decreasing the applied field each time by a fixed amount, until a negative field of magnitude $\boldsymbol{H}_{0}$ is reached. In the hysteresis loop, we plot the average equilibrium magnetization as a function of the applied field. In our example, we consider $\boldsymbol{H}_{0}=600 \mathrm{Oe}$ in the $x$ direction.

Typically, in a multilayer, the magnetization can be found in one of two states: The $\mathrm{S}$ state, and the $\mathrm{C}$ state. The magnetization reversal process associated to the $\mathrm{S}$ state usually occurs by a rotation of the magnetization in the interior of the domain, followed by the appearance of boundary layers, which can be removed if the applied field is strong enough. In the $\mathrm{C}$ state, the magnetization reversal process occurs by nucleating a magnetic vortex, which is subsequently expelled.

In Figure 4, we plot hysteresis loops associated with a double layer initialized with S states in each layer, and loops associated with a double layer with $\mathrm{C}$ states in each layer. For the given dimensions, and in the absence of spin currents, a magnetic field of -3000 is required to switch the magnetization of the top layer when both layers are in the $\mathrm{S}$ state. When both layers are in the $\mathrm{C}$ state, in the absence of spin currents, a vortex nucleates at approximately 1000e. A field of approximately $-100 \mathrm{Oe}$ is required to expel the vortex, and successfully reverse the magnetization of the bottom layer. Due to thickness difference, the bottom layer is easier to be switched, which requires a weaker external field $(-100$ Oe instead of $-300 \mathrm{Oe})$.

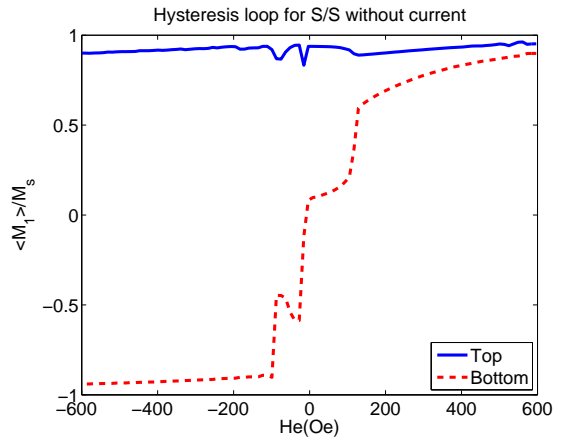

(a) $\mathrm{S} / \mathrm{S}$

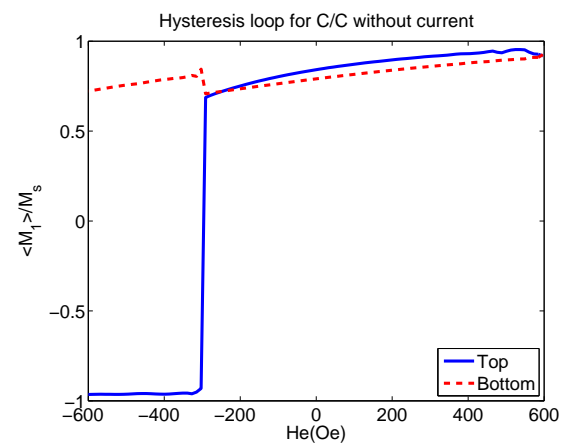

(b) $\mathrm{C} / \mathrm{C}$

FIgURE 4. Hysteresis loops for a multilayer with thicknesses $D_{1}=60 \mathrm{~nm}, D_{2}=10 \mathrm{~nm}$, and $D_{3}=20 \mathrm{~nm}$ and without spin currents: (a): S/S; (b): C/C.

Figure 5 shows hysteresis loops simulated by (38) when $\boldsymbol{j}_{n}=15 \times 10^{10} \mathrm{~A} / \mathrm{m}^{2}$ and $\tau / \tau_{J}=1$. Similar phenomena are observed for a smaller $\boldsymbol{j}_{n}$ and 
$\tau / \tau_{J}=0$. Magnetization of the bottom layer initially tends to be a vortex state and magnetization of the top layer is still uniaxially. Accumulation of spin transfer torque eventually makes the magnetization reversal of the bottom layer happen.

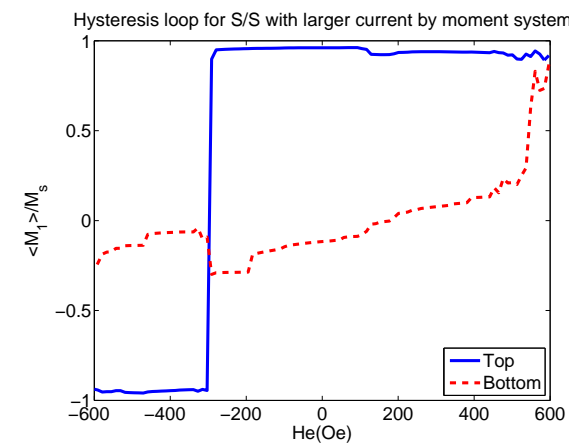

(a) $\mathrm{S} / \mathrm{S}$

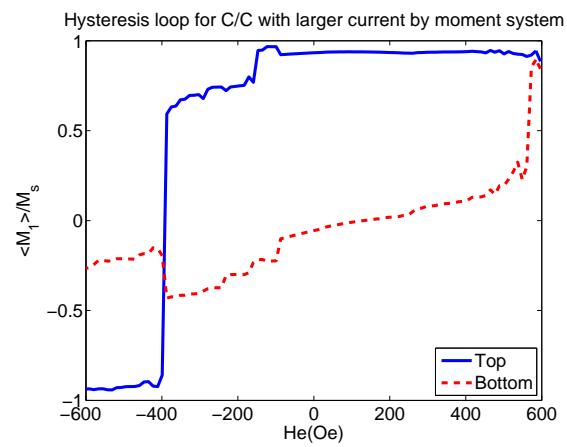

(b) $\mathrm{C} / \mathrm{C}$

FiguRE 5. Hysteresis loops for a multilayer with thicknesses $D_{1}=60 \mathrm{~nm}, D_{2}=10 \mathrm{~nm}$, and $D_{3}=20 \mathrm{~nm}$ and with larger spin currents by Equation (38): (a): S/S; (b): C/C.

From Figure 4, the bottom layer is easy to be switched. Moreover, spin current is applied from the bottom layer, which contributes to the effective field. Therefore, magnetization of the bottom layer initially tends to be a vortex state and magnetization of the top layer is still uniaxially. Spin current acts as a torque for the top layer, so the magnetization reversal happens in the top layer after the accumulation of spin transfer torque is strong enough.

When $\boldsymbol{j}_{n}=10 \times 10^{10} \mathrm{~A} / \mathrm{m}^{2}$ is applied from the top layer, magnetization of both layers is plotted in Figure 6 for $\tau / \tau_{J}=1$. Spin current is applied from the top layer, which contributes to the effective field, so magnetization reversal of the top layer happens at first since this layer is only $20 \mathrm{~nm}$ thick, 300 Oe for both $\mathrm{S} / \mathrm{S}$ and $\mathrm{C} / \mathrm{C}$ states. Magnetization of the bottom layer is still uniaxially since spin transfer torque is not strong enough. Afterwards, spin transfer torque tends to drive magnetization of the bottom layer escape from its initial state. A vortex state is observed since the bottom layer is $60 \mathrm{~nm}$ thick. Eventually, magnetization reversal of the bottom layer happens around -600 Oe. Maximum GMR can be easily achieved in this case (300Oe).

4.3. Magnetic reversal for information manipulation and storage. Based on the above results, we propose a possible procedure to operate magnetization of both top and bottom layers for the purpose of information manipulation and storage:

Step 0 : Initialize the device with $\mathrm{S} / \mathrm{S}$ states for $5 \mathrm{~ns}$. 


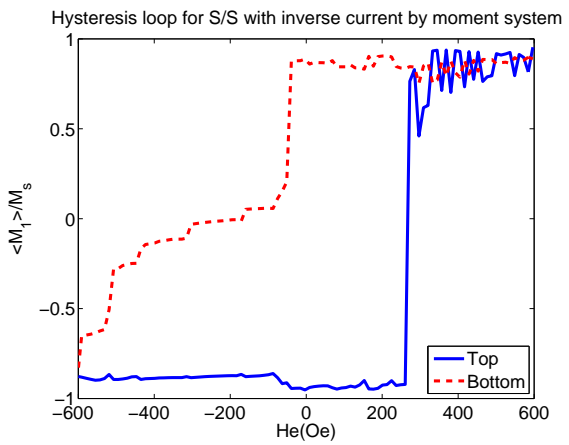

(a) $\mathrm{S} / \mathrm{S}$

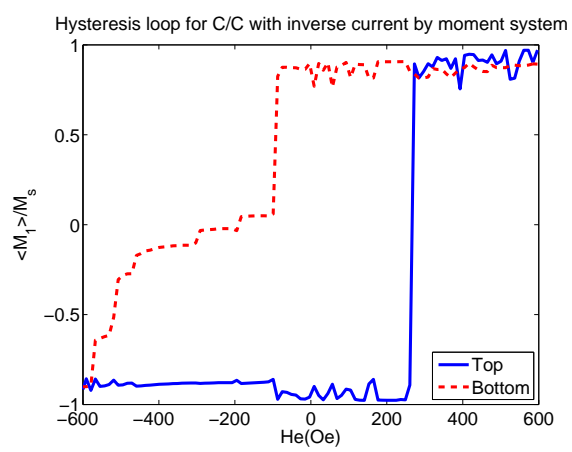

(b) $\mathrm{C} / \mathrm{C}$

FiguRE 6. Hysteresis loops for a multilayer with thicknesses $D_{1}=60 \mathrm{~nm}, D_{2}=10 \mathrm{~nm}$, and $D_{3}=20 \mathrm{~nm}$ and with inverse spin currents by Equation (38): (a): S/S; (b): C/C.

Step 1 : Apply external magnetic field with strength 6000e, and current $\boldsymbol{j}_{n}=15 \times 10^{10} \mathrm{~A} / \mathrm{m}^{2}$ from the bottom layer based on Figure 5 for 5 ns.

Step 2 : Remove all external fields and let the device relax for 5ns.

Step 3 : Apply external magnetic field with strength 3000e, and current $\boldsymbol{j}_{n}=10 \times 10^{10} \mathrm{~A} / \mathrm{m}^{2}$ from the top layer based on Figure 6 for $5 \mathrm{~ns}$.

Step 4 : Remove all external fields and let the device relax for 5ns.

Step 5 : Repeat Steps 1 to 4 to check the reproducibility of above reversals.

Figure 7 shows the magnetization of above steps. Magnetization of top and bottom layers is antiparallel in Step 0, parallel in Step 1 which is also stable as verified in Step 2, antiparallel in Step 3 which is stable as verified in Step 4. This process is reproduceable as verified in Step 5.

\section{Conclusion}

We have derived a macroscopic model to describe the spin dynamics starting from a Schrödinger equation in the spinor form using the Wigner transform and a moment closure. This model, together with the magnetization dynamics described by the Landau-Lifshitz-Gilbert equation, gives a full description of the magnetization in ferromagnetic multilayers in the presence of spin currents. Moreover, this model recovers the model in [42] when the spin-magnetization coupling is weak. Its validation is analyzed and shown by several examples. Successful application of our model (34)(36) to current-driven domain wall motions has also been conducted with quantitative agreements with experimental data [12].

From a modeling viewpoint, we stress that our approach produces a connection from quantum mechanics (1), to the Boltzmann equation (12), and then to the moment system (26)-(29), and finally to the drift-diffusion equation (34)-(36). Although only the drift-diffusion equation has been examined carefully, other models, such as the moment system and the Boltzmann 


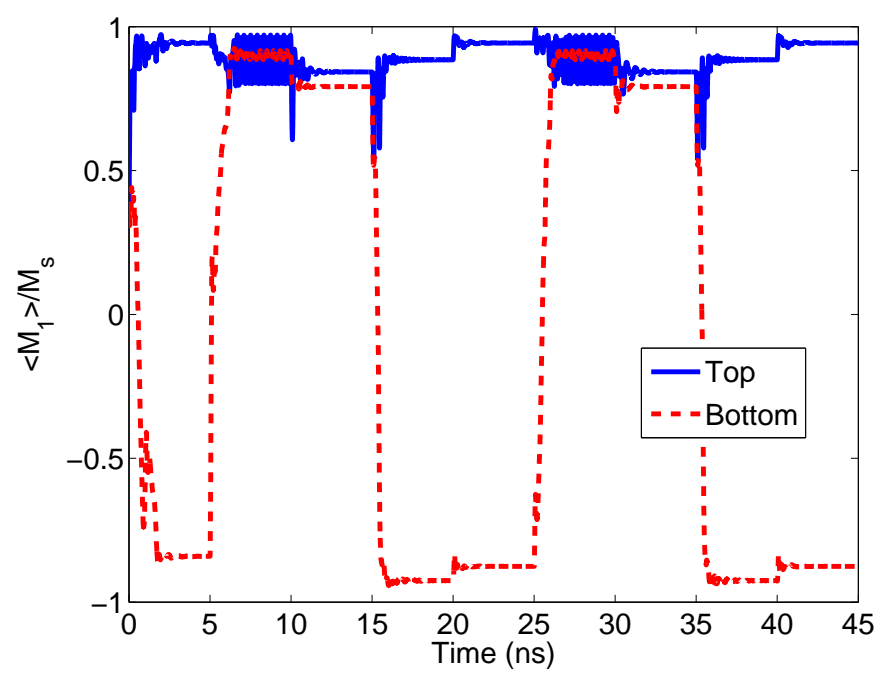

FigURE 7. A sequence of magnetization reversals for manipulation and storage of information.

equation, could be helpful to understand experimental results in other scenarios. For example, femtosecond spin dynamics in experiments [30] cannot be well described by the drift-diffusion equation (34)-(36), and the moment system (26)-(29) shall play an important role in this case.

\section{ACKNOWLEDGMENTS}

CJGC acknowledges support from the National Science Foundation via grants DMS-0645766 and DMS-1065942. XY acknowledges support from the National Science Foundation via grant DMS-1418936 and the Regents Junior Faculty Fellowship of the University of California, Santa Barbara. This work was also partially supported by KI-Net NSF RNMS grant No. 1107444 . 


\section{ApPendix A}

In this Appendix, we give the derivation of (9) from (1) by the Wigner transform (3) in details. Plugging (1) into (8) produces

$$
\begin{array}{rl}
\mathrm{i} \hbar \partial_{t} & W(\boldsymbol{x}, \boldsymbol{v}, t)=\left(\frac{m}{2 \pi \hbar}\right)^{3} \int_{\mathbb{R}^{3}}\left(-\frac{\hbar^{2}}{2 m} \nabla_{\boldsymbol{x}}^{2} \boldsymbol{\psi}\left(\boldsymbol{x}-\frac{\boldsymbol{y}}{2}, t\right) \otimes \boldsymbol{\psi}^{*}\left(\boldsymbol{x}+\frac{\boldsymbol{y}}{2}, t\right) \mathrm{e}^{\mathrm{i} \frac{m}{\hbar} \boldsymbol{v} \cdot \boldsymbol{y}}\right. \\
& \left.+\boldsymbol{\psi}\left(\boldsymbol{x}-\frac{\boldsymbol{y}}{2}, t\right) \otimes\left(\frac{\hbar^{2}}{2 m} \nabla_{\boldsymbol{x}}^{2} \boldsymbol{\psi}^{*}\left(\boldsymbol{x}+\frac{\boldsymbol{y}}{2}, t\right)\right) \mathrm{e}^{\mathrm{i} \frac{m}{\hbar} \boldsymbol{v} \cdot \boldsymbol{y}}\right) \mathrm{d} \boldsymbol{y} \\
& +\left(\frac{m}{2 \pi \hbar}\right)^{3} \int_{\mathbb{R}^{3}}\left(\left(V\left(\boldsymbol{x}-\frac{\boldsymbol{y}}{2}\right) \boldsymbol{\psi}\left(\boldsymbol{x}-\frac{\boldsymbol{y}}{2}, t\right)\right) \otimes \boldsymbol{\psi}^{*}\left(\boldsymbol{x}+\frac{\boldsymbol{y}}{2}, t\right) \mathrm{e}^{\mathrm{i} \frac{m}{\hbar} \boldsymbol{v} \cdot \boldsymbol{y}}\right. \\
& \left.+\boldsymbol{\psi}\left(\boldsymbol{x}-\frac{\boldsymbol{y}}{2}, t\right) \otimes\left(-V\left(\boldsymbol{x}+\frac{\boldsymbol{y}}{2}\right) \boldsymbol{\psi}^{*}\left(\boldsymbol{x}+\frac{\boldsymbol{y}}{2} \boldsymbol{y}, t\right)\right) \mathrm{e}^{\mathrm{i} \frac{m}{\hbar} \boldsymbol{v} \cdot \boldsymbol{y}}\right) \mathrm{d} \boldsymbol{y} \\
& -\left(\frac{m}{2 \pi \hbar}\right)^{3} \int_{\mathbb{R}^{3}}\left(\left(\frac{J}{2} \hat{\boldsymbol{\sigma}} \cdot \boldsymbol{m}\left(\boldsymbol{x}-\frac{\boldsymbol{y}}{2}, t\right) \boldsymbol{\psi}\left(\boldsymbol{x}-\frac{\boldsymbol{y}}{2}, t\right)\right) \otimes \boldsymbol{\psi}^{*}\left(\boldsymbol{x}+\frac{\boldsymbol{y}}{2}, t\right) \mathrm{e}^{\mathrm{i} \frac{m}{\hbar} \boldsymbol{v} \cdot \boldsymbol{y}}\right. \\
& \left.+\boldsymbol{\psi}\left(\boldsymbol{x}-\frac{\boldsymbol{y}}{2}, t\right) \otimes\left(-\boldsymbol{\psi}^{*}\left(\boldsymbol{x}+\frac{\boldsymbol{y}}{2}, t\right) \frac{J}{2} \hat{\boldsymbol{\sigma}} \cdot \boldsymbol{m}\left(\boldsymbol{x}+\frac{\boldsymbol{y}}{2}, t\right)\right) \mathrm{e}^{\mathrm{i} \frac{m}{\hbar} \boldsymbol{v} \cdot \boldsymbol{y}}\right) \mathrm{d} \boldsymbol{y} .
\end{array}
$$

The equation (9) is obtained by simplifying the above three integrals individually as described below.

Noticing that

$$
\begin{array}{r}
\nabla_{\boldsymbol{x}} \boldsymbol{\psi}\left(\boldsymbol{x}-\frac{\boldsymbol{y}}{2}, t\right)=-2 \nabla_{\boldsymbol{y}} \boldsymbol{\psi}\left(\boldsymbol{x}-\frac{\boldsymbol{y}}{2}, t\right), \\
\nabla_{\boldsymbol{x}} \boldsymbol{\psi}\left(\boldsymbol{x}+\frac{\boldsymbol{y}}{2}, t\right)=2 \nabla_{\boldsymbol{y}} \boldsymbol{\psi}\left(\boldsymbol{x}+\frac{\boldsymbol{y}}{2}, t\right)
\end{array}
$$

brings

$\nabla_{\boldsymbol{y}}^{2} \boldsymbol{\psi} \otimes \boldsymbol{\psi}^{*} \mathrm{e}^{\mathrm{i} \frac{m}{\hbar} \boldsymbol{v} \cdot \boldsymbol{y}}=\nabla_{\boldsymbol{y}} \cdot\left(\nabla_{\boldsymbol{y}} \boldsymbol{\psi} \otimes \boldsymbol{\psi}^{*} \mathrm{e}^{\mathrm{i} \frac{m}{\hbar} \boldsymbol{v} \cdot \boldsymbol{y}}\right)-\nabla_{\boldsymbol{y}} \boldsymbol{\psi} \otimes \cdot \nabla_{\boldsymbol{y}} \boldsymbol{\psi}^{*} \mathrm{e}^{\mathrm{i} \frac{m}{\hbar} \boldsymbol{v} \cdot \boldsymbol{y}}-\mathrm{i} \frac{m}{\hbar} \boldsymbol{v} \cdot \nabla_{\boldsymbol{y}} \boldsymbol{\psi} \otimes \boldsymbol{\psi}^{*} \mathrm{e}^{\mathrm{i} \frac{m}{\hbar} \boldsymbol{v} \cdot \boldsymbol{y}}$, $\boldsymbol{\psi} \otimes \nabla_{\boldsymbol{y}}^{2} \boldsymbol{\psi}^{*} \mathrm{e}^{\mathrm{i} \frac{m}{\hbar} \boldsymbol{v} \cdot \boldsymbol{y}}=\nabla_{\boldsymbol{y}} \cdot\left(\boldsymbol{\psi} \otimes \nabla_{\boldsymbol{y}} \boldsymbol{\psi}^{*} \mathrm{e}^{\mathrm{i} \frac{m}{\hbar} \boldsymbol{v} \cdot \boldsymbol{y}}\right)-\nabla_{\boldsymbol{y}} \boldsymbol{\psi} \otimes \cdot \nabla_{\boldsymbol{y}} \boldsymbol{\psi}^{*} \mathrm{e}^{\mathrm{i} \frac{m}{\hbar} \boldsymbol{v} \cdot \boldsymbol{y}}-\mathrm{i} \frac{m}{\hbar} \boldsymbol{v} \cdot \boldsymbol{\psi} \otimes \nabla_{\boldsymbol{y}} \boldsymbol{\psi}^{*} \mathrm{e}^{\mathrm{i} \frac{m}{\hbar} \boldsymbol{v} \cdot \boldsymbol{y}}$,

where the first $\boldsymbol{\psi}$ acts on the variable $\boldsymbol{x}-\frac{\boldsymbol{y}}{2}$ and the second $\boldsymbol{\psi}$ acts on the variable $\boldsymbol{x}+\frac{\boldsymbol{y}}{2}$. 
The divergence theorem along with the fact that $\boldsymbol{\psi}, \boldsymbol{\psi}^{*}$ vanish at $\pm \infty$ simplifies the first integral as

$$
\begin{aligned}
& \left(\frac{m}{2 \pi \hbar}\right)^{3} \int_{\mathbb{R}^{3}} 2 \hbar \mathrm{i} \boldsymbol{v} \cdot\left(\nabla_{\boldsymbol{y}} \boldsymbol{\psi}\left(\boldsymbol{x}-\frac{\boldsymbol{y}}{2}, t\right) \otimes \boldsymbol{\psi}^{*}\left(\boldsymbol{x}+\frac{\boldsymbol{y}}{2}, t\right)\right. \\
& \left.\quad-\boldsymbol{\psi}\left(\boldsymbol{x}-\frac{\boldsymbol{y}}{2}, t\right) \otimes \nabla_{\boldsymbol{y}} \boldsymbol{\psi}^{*}\left(\boldsymbol{x}+\frac{\boldsymbol{y}}{2}, t\right)\right) \mathrm{e}^{\mathrm{i} \frac{m}{\hbar} \boldsymbol{v} \cdot \boldsymbol{y}} \mathrm{d} \boldsymbol{y} \\
& \quad=\left(\frac{m}{2 \pi \hbar}\right)^{3} \int_{\mathbb{R}^{3}} \mathrm{i} \hbar \boldsymbol{v} \cdot\left(-\nabla_{\boldsymbol{x}} \boldsymbol{\psi}\left(\boldsymbol{x}-\frac{\boldsymbol{y}}{2}, t\right) \otimes \boldsymbol{\psi}^{*}\left(\boldsymbol{x}+\frac{\boldsymbol{y}}{2}, t\right)\right. \\
& \left.\quad-\boldsymbol{\psi}\left(\boldsymbol{x}-\frac{\boldsymbol{y}}{2}, t\right) \otimes \nabla_{\boldsymbol{x}} \boldsymbol{\psi}^{*}\left(\boldsymbol{x}+\frac{\boldsymbol{y}}{2}, t\right)\right) \mathrm{e}^{\mathrm{i} \frac{m}{\hbar} \boldsymbol{v} \cdot \boldsymbol{y}} \mathrm{d} \boldsymbol{y} \\
& \quad=-i \hbar \boldsymbol{v} \cdot \nabla_{\boldsymbol{x}}\left(\left(\frac{m}{2 \pi \hbar}\right)^{3} \int_{\mathbb{R}^{3}} \boldsymbol{\psi}\left(\boldsymbol{x}-\frac{\boldsymbol{y}}{2}, t\right) \otimes \boldsymbol{\psi}^{*}\left(\boldsymbol{x}+\frac{\boldsymbol{y}}{2}, t\right) \mathrm{e}^{\mathrm{i} \frac{m}{\hbar} \boldsymbol{v} \cdot \boldsymbol{y}} \mathrm{d} \boldsymbol{y}\right) \\
& \quad=-i \hbar \boldsymbol{v} \cdot \nabla_{\boldsymbol{x}} W(\boldsymbol{x}, \boldsymbol{v}, t) .
\end{aligned}
$$

Taylor expansion gives

$$
\begin{aligned}
& V\left(\boldsymbol{x}-\frac{\boldsymbol{y}}{2}\right)=V(\boldsymbol{x})-\frac{1}{2} \boldsymbol{y} \cdot \nabla_{\boldsymbol{x}} V(\boldsymbol{x})+\mathcal{O}\left(\left(\frac{1}{2}\right)^{2}\right), \\
& V\left(\boldsymbol{x}+\frac{\boldsymbol{y}}{2}\right)=V(\boldsymbol{x})+\frac{1}{2} \boldsymbol{y} \cdot \nabla_{\boldsymbol{x}} V(\boldsymbol{x})+\mathcal{O}\left(\left(\frac{1}{2}\right)^{2}\right),
\end{aligned}
$$

which simplifies the second integral as

$$
\begin{aligned}
& \left(\frac{m}{2 \pi \hbar}\right)^{3} \int_{\mathbb{R}^{3}} \boldsymbol{y} \cdot(-e \boldsymbol{E}) \boldsymbol{\psi}\left(\boldsymbol{x}-\frac{\boldsymbol{y}}{2}, t\right) \otimes \boldsymbol{\psi}^{*}\left(\boldsymbol{x}+\frac{\boldsymbol{y}}{2}, t\right) \mathrm{e}^{\mathrm{i} \frac{m}{\hbar} \boldsymbol{v} \cdot \boldsymbol{y}} \mathrm{d} \boldsymbol{y} \\
& \quad=\left(\frac{m}{2 \pi \hbar}\right)^{3} \int_{\mathbb{R}^{3}} \boldsymbol{\psi}\left(\boldsymbol{x}-\frac{\boldsymbol{y}}{2}, t\right) \otimes \boldsymbol{\psi}^{*}\left(\boldsymbol{x}+\frac{\boldsymbol{y}}{2}, t\right) \frac{\hbar}{\mathrm{i} m}(-e \boldsymbol{E}) \cdot \nabla_{\boldsymbol{v}} \mathrm{e}^{\mathrm{i} \frac{m}{\hbar} \boldsymbol{v} \cdot \boldsymbol{y}} \mathrm{d} \boldsymbol{y} \\
& \quad=\frac{\mathrm{i} e \hbar}{m} \boldsymbol{E} \cdot \nabla_{\boldsymbol{v}} W(\boldsymbol{x}, \boldsymbol{v}, t),
\end{aligned}
$$

where $\boldsymbol{E}=\nabla V / e$. Similarly,

$$
\begin{aligned}
& \boldsymbol{m}\left(\boldsymbol{x}-\frac{\boldsymbol{y}}{2}, t\right)=\boldsymbol{m}(\boldsymbol{x})-\frac{1}{2} \boldsymbol{y} \cdot \nabla_{\boldsymbol{x}} \boldsymbol{m}(\boldsymbol{x})+\mathcal{O}\left(\left(\frac{1}{2}\right)^{2}\right) \\
& \boldsymbol{m}\left(\boldsymbol{x}+\frac{\boldsymbol{y}}{2}, t\right)=\boldsymbol{m}(\boldsymbol{x})+\frac{1}{2} \boldsymbol{y} \cdot \nabla_{\boldsymbol{x}} \boldsymbol{m}(\boldsymbol{x})+\mathcal{O}\left(\left(\frac{1}{2}\right)^{2}\right)
\end{aligned}
$$


which simplifies the third integral as

$$
\begin{aligned}
- & \left(\frac{m}{2 \pi \hbar}\right)^{3} \int_{\mathbb{R}^{3}}\left(\left(\frac{J}{2} \hat{\boldsymbol{\sigma}} \cdot \boldsymbol{m}(\boldsymbol{x}, t) \boldsymbol{\psi}\left(\boldsymbol{x}-\frac{\boldsymbol{y}}{2}, t\right)\right) \otimes \boldsymbol{\psi}^{*}\left(\boldsymbol{x}+\frac{\boldsymbol{y}}{2}, t\right) \mathrm{e}^{\mathrm{i} \frac{m}{\hbar} \boldsymbol{v} \cdot \boldsymbol{y}}\right. \\
& \left.+\boldsymbol{\psi}\left(\boldsymbol{x}-\frac{\boldsymbol{y}}{2}, t\right) \otimes\left(-\boldsymbol{\psi}^{*}\left(\boldsymbol{x}+\frac{\boldsymbol{y}}{2}, t\right) \frac{J}{2} \hat{\boldsymbol{\sigma}} \cdot \boldsymbol{m}(\boldsymbol{x}, t)\right) \mathrm{e}^{\mathrm{i} \frac{m}{\hbar} \boldsymbol{v} \cdot \boldsymbol{y}}\right) \mathrm{d} \boldsymbol{y} \\
& =-\left(\frac{J}{2} \hat{\boldsymbol{\sigma}} \cdot \boldsymbol{m}(\boldsymbol{x}, t) W(\boldsymbol{x}, \boldsymbol{v}, t)-W(\boldsymbol{x}, \boldsymbol{v}, t) \frac{J}{2} \hat{\boldsymbol{\sigma}} \cdot \boldsymbol{m}(\boldsymbol{x}, t)\right) \\
& =-\left[\frac{J}{2} \hat{\boldsymbol{\sigma}} \cdot \boldsymbol{m}(\boldsymbol{x}, t), W(\boldsymbol{x}, \boldsymbol{v}, t)\right] .
\end{aligned}
$$

Combining above three terms, we get (9).

\section{REFERENCES}

1. A. Arnold, Self-consistent relaxation time models in quantum mechanics, Comm. PDE 21 (1996), 473-506.

2. A. Arnold, I. Gamba, M. P. Gualdani, S. Mischler, C. Mouhot, and C. Sparber, The Wigner-Fokker-Planck equation: stationary state and large time behavior, Math. Model Methods Appl. Sci. 22 (2012), no. 11, 1250034.

3. A. Arnold, J. L. López, P. Markowich, and J. Soler, An analysis of quantum FokkerPlanck models: A Wigner function approach, Rev. Mat. Iberoamericana 20 (2004), $771-814$.

4. V.E. Badalassi, H.D. Ceniceros, and S. Banerjee, Computation of multiphase systems with phase field models, J. Comp. Phys. 190 (2003), no. 2, 371 - 397.

5. M. N. Baibich, J. M. Broto, A. Fert, F. Nguyen Van Dau, F. Petroff, P. Etienne, G. Creuzet, A. Friederich, and J. Chazelas, Giant magnetoresistance of (001)Fe/(001)Cr magnetic superlattices, Phys. Rev. Lett. 61 (1988), 2472-2475.

6. C. Bardos, L. Erdös, F. Golse, N. Mauser, and H.-T. Yau, Derivation of the Schrödinger-Poisson equation from the quantum N-body problem, C. R. Math. Acad. Sci. Paris 334 (2002), 515-520.

7. C. Bardos, F. Golse, and C. D. Levermore, The acoustic limit for the Boltzmann equation, Arch. Ration. Mech. Anal. 153 (2000), 177-204.

8. L. Berger, Emission of spin waves by a magnetic multilayer traversed by a current, Phys. Rev. B 54 (1996), 9353-9358.

9. A. Brataas, A. D. Kent, and H. Ohno, Current-induced torques in magnetic materials, Nat. Mater. 11 (2012), 372-381.

10. C. Cercignani, I. Gamba, J. Jerome, and C.-W. Shu, Device benchmark comparison$s$ via kinetic, hydrodynamic, and high-field models, Comput. Methods Appl. Mech. Engrg. 181 (2000), 381-392.

11. C. Cercignani, I.M. Gamba, and C.D. Levermore, High field approximations to a Boltzmann-Poisson system and boundary conditions in a semiconductor, Appl. Math. Lett. 10 (1997), $111-117$.

12. J. Chen, C. J. García-Cervera, and X. Yang, Mean-field dynamics of the spinmagnetization coupling in ferromagnetic materials: Application to current-driven domain wall motions, submitted (2015).

13. R. Cheng and Q. Niu, Microscopic derivation of spin-transfer torque in ferromagnets, Phys. Rev. B 88 (2013), 024422.

14. J. J. Douglas and T. Dupont, Alternating-direction galerkin methods on rectangles, SYNSPADE-1970, Numerical Solution of Partial Differential Equations-II (B. Hubbard, ed.), Academic Press, New York, 1971, pp. 133 - 213. 
15. S. Emori, U. Bauer, S.-M. Ahn, E. Martinez, and G. S. D. Beach, Current-driven dynamics of chiral ferromagnetic domain walls, Nat. Mater. 12 (2013), 611-616.

16. F. Filbet and S. Jin, A class of asymptotic-preserving schemes for kinetic equations and related problems with stiff sources, J. Comp. Phys. 229 (2010), no. 20, 7625 7648.

17. C. J. García-Cervera and X.-P. Wang, Spin-polarized currents in ferromagnetic multilayers, J. Comput. Phys. 224 (2007), no. 2, 699-711. MR 2330291 (2008c:82087)

18. G. D. Gaspari, Bloch equation for conduction-electron spin resonance, Phys. Rev. 151 (1966), 215-219.

19. T.L. Gilbert, Phys. Rev. 100 (1955), 1243, [Abstract only; full report, Armor Research Foundation Project No. A059, Supplementary Report, May 1, 1956 (unpublished)].

20. F. Golse and L. Saint-Raymond, The Navier-Stokes limit of the Boltzmann equation for bounded collision kernels, Invent. Math. 155 (2004), 81-161.

21. P. Grünberg, R. Schreiber, Y. Pang, M. B. Brodsky, and H. Sowers, Layered magnetic structures: Evidence for antiferromagnetic coupling of Fe layers across Cr interlayers, Phys. Rev. Lett. 57 (1986), 2442-2445.

22. R. Hanson, F. M. Mendoza, R. J. Epstein, and D. D. Awschalom, Polarization and readout of coupled single spins in diamond, Phys. Rev. Lett. 97 (2006), 087601.

23. T. Hillen, Hyperbolic models for chemosensitive movement, Math. Models and Methods in Appl. Sci. 12 (2002), 1007-1034.

24. I. Žutić, J. Fabian, and S. Das Sarma, Spintronics: Fundamentals and applications, Rev. Mod. Phys. 76 (2004), 323-410.

25. J. I. Kaplan, Diffusion constant in the effective Bloch equation for ferromagnetic resonance in metals, Phys. Rev. 143 (1966), 351-352.

26. L. Landau and E. Lifshitz, On the theory of the dispersion of magnetic permeability in ferromagnetic bodies, Physikalische Zeitschrift der Sowjetunion 8 (1935), 153-169.

27. C. D. Levermore, Moment closure hierarchies for kinetic theories, J. Stat. Phys. 83 (1996), no. 5-6, 1021-1065.

28. P. Markowich, T. Paul, and C. Sparber, Bohmian measures and their classical limit, Journal of Functional Analysis 259 (2010), 1542 - 1576.

29. P. A. Markowich and N. J. Mauser, The classical limit of a self-consistent quantumVlasov equation in 3d, Math. Model Methods Appl. Sci. 3 (1993), no. 1, 109-124.

30. J. Mendil, P. Nieves, O. Chubykalo-Fesenko, J. Walowski, T. Santos, S. Pisana, and M. Mnzenberg, Resolving the role of femtosecond heated electrons in ultrafast spin dynamics, Scientific Reports 4 (2014), 3980.

31. I. M. Miron, T. Moore, H. Szambolics, L. D. Buda-Prejbeanu, S. Auffret, B. Rodmacq, S. Pizzini, J. Vogel, M. Bonfim, A. Schuhl, and G. Gaudin, Fast current-induced domain-wall motion controlled by the Rashba effect, Nat. Mater. 10 (2011), 419-423.

32. T. Ono, H. Miyajima, K. Shigeto, K. Mibu, N. Hosoito, and T. Shinjo, Propagation of a magnetic domain wall in a submicrometer magnetic wire, Science 284 (1999), no. 5413, 468-470.

33. F. Piéchon and A. Thiaville, Spin transfer torque in continuous textures: Semiclassical Boltzmann approach, Phys. Rev. B 75 (2007), 174414.

34. Y. Qi and S. Zhang, Spin diffusion at finite electric and magnetic fields, Phys. Rev. B 67 (2003), 052407.

35. D.C. Ralph and M.D. Stiles, Spin transfer torques, J. Magn. Magn. Mater. 320 (2008), no. $7,1190-1216$

36. S. Sanvito, Organic spintronics: Filtering spins with molecules, Nat. Mater. 10 (2011), 484-485.

37. A. Shpiro, P. M. Levy, and S. Zhang, Self-consistent treatment of nonequilibrium spin torques in magnetic multilayers, Phys. Rev. B 67 (2003), 104430.

38. J.C. Slonczewski, Current-driven excitation of magnetic multilayers, J. Magn. Magn. Mat. 159 (1996), L1-L7. 
39. C. Sparber and P. Markowich, Semiclassical asymptotics for the MaxwellDirac system, J. Math. Phys. 44 (2003), 4555-4572.

40. G. Tatara and H. Kohno, Theory of current-driven domain wall motion: Spin transfer versus momentum transfer, Phys. Rev. Lett. 92 (2004), 086601.

41. X.-P. Wang, C. J. García-Cervera, and W. E, A Gauss-Seidel projection method for the Landau-Lifshitz equation, J. Comp. Phys. 171 (2001), 357-372.

42. S. Zhang, P. M. Levy, and A. Fert, Mechanisms of spin-polarized current-driven magnetization switching, Phys. Rev. Lett. 88 (2002), 236601.

43. S. Zhang and Z. Li, Roles of nonequilibrium conduction electrons on the magnetization dynamics of ferromagnets, Phys. Rev. Lett. 93 (2004), 127204.

Mathematics Department, South Hall 6705, University of California, Santa BARBARA, CA93106, USA

E-mail address: cjr@math.ucsb.edu

Mathematics Department, South Hall 6707, University of California, Santa BARBARA, CA93106, USA

E-mail address: cgarcia@math.ucsb.edu

Mathematics Department, South Hall 6709, University of California, Santa BARBARA, CA93106, USA

E-mail address: xuyang@math.ucsb.edu 